OPEN ACCESS

Edited by: Kyoko Imanaka-Yoshida, Mie University, Japan

Reviewed by: Shizuya Saika, Wakayama Medical University Hospital, Japan Hideki Kanamaru, Mie University, Japan

*Correspondence: Pavle R. Andjus pandjus@bio.bg.ac.rs Melitta Schachner schachner@dls.rutgers.edu

Specialty section: This article was submitted to Inflammation,

a section of the journal

Frontiers in Immunology

Received: 31 October 2020 Accepted: 31 December 2020 Published: 19 February 2021

Citation:

Bijelić $D$, Adžić $M$, Perić $M$, Jakovčevski I, Förster E, Schachner $M$ and Andjus PR (2021) Different

Functions of Recombinantly Expressed Domains of Tenascin-C in Glial Scar Formation.

Front. Immunol. 11:624612. doi: 10.3389/fimmu.2020.624612

\section{Different Functions of Recombinantly Expressed Domains of Tenascin-C in Glial Scar Formation}

\author{
Dunja Bijelić ${ }^{1}$, Marija Adžić ${ }^{1}$, Mina Perić ${ }^{1}$, Igor Jakovčevski ${ }^{2}$, Eckart Förster ${ }^{2}$, \\ Melitta Schachner ${ }^{3 *}$ and Pavle R. Andjus ${ }^{1 *}$ \\ ${ }^{1}$ Centre for Laser Microscopy, Faculty of Biology, Institute of Physiology and Biochemistry "Jean Giaja", University of Belgrade, \\ Belgrade, Serbia, 2 Institut für Neuroanatomie und Molekulare Hirnforschung, Ruhr-Universität Bochum, Bochum, Germany, \\ ${ }^{3}$ Keck Center for Collaborative Neuroscience and Department of Cell Biology and Neuroscience, Rutgers University, \\ Piscataway, NJ, United States
}

Extracellular matrix glycoprotein tenascin- $\mathrm{C}(\mathrm{TnC})$ is highly expressed in vertebrates during embryonic development and thereafter transiently in tissue niches undergoing extensive remodeling during regeneration after injury. TnC's different functions can be attributed to its multimodular structure represented by distinct domains and alternatively spliced isoforms. Upon central nervous system injury, TnC is upregulated and secreted into the extracellular matrix mainly by astrocytes. The goal of the present study was to elucidate the role of different $\mathrm{TnC}$ domains in events that take place after spinal cord injury (SCI). Astrocyte cultures prepared from $\mathrm{TnC}$-deficient (TnC-/-) and wild-type $(\mathrm{TnC}+/+)$ mice were scratched and treated with different recombinantly generated TnC fragments. Gap closure, cell proliferation and expression of GFAP and cytokines were determined in these cultures. Gap closure in vitro was found to be delayed by TnC fragments, an effect mainly mediated by decreasing proliferation of astrocytes. The most potent effects were observed with fragments FnD, FnA and their combination. TnC-/- astrocyte cultures exhibited higher GFAP protein and mRNA expression levels, regardless of the type of fragment used for treatment. Application of TnC fragments induced also pro-inflammatory cytokine production by astrocytes in vitro. In vivo, however, the addition of FnD or Fn(D+A) led to a difference between the two genotypes, with higher levels of GFAP expression in $\mathrm{TnC}+/+$ mice. FnD treatment of injured TnC-/- mice increased the density of activated microglia/macrophages in the injury region, while overall cell proliferation in the injury site was not affected. We suggest that altogether these results may explain how the reaction of astrocytes is delayed while their localization is restricted to the border of the injury site to allow microglia/macrophages to form a lesion core during the first stages of glial scar formation, as mediated by $\mathrm{TnC}$ and, in particular, the alternatively spliced FnD domain.

Keywords: astrocyte, glial scar, microglia/macrophages, spinal cord injury, tenascin-C 


\section{INTRODUCTION}

Spinal cord injury (SCI) is a severe neurological disorder with a limited hope for recovery, thus presenting a health care and socioeconomic problem. SCI is a two-step process, primary mechanical injury is followed by secondary inflammation and apoptosis through which existing injury spreads further into the surrounding tissue (1). The hallmark of these events is the formation of the glial scar with two distinct parts. The lesion core is formed by stromal fibroblasts and inflammatory immune cells, while hypertrophic astrocytes demarcate the lesion border (2). The role of the glial scar has been often discussed, once seen as a barrier to complete regeneration (3), but often regarded as important for axonal regrowth and as the source of growth factors and other permissive molecules (4). A majority of cellular functions, signal transduction, and tissue homeostasis are maintained by extracellular matrix components (ECM) making the ECM an interesting target for modulation of the outcome of injury. It is noteworthy in this context that the ECM glycoprotein tenascin-C $(\mathrm{TnC})$ is strongly upregulated after injury of central nervous system (5).

Tenascin-C $(\mathrm{TnC})$ is a large multimodular glycoprotein with a hexabrahion structure (6). Each arm contains four distinct domains: amino-terminal tenascin assembly (TA) domain, epidermal growth factor-like (EGFL) repeats, fibronectin type III (FnIII) domains, and a globular fibrinogen-homology domain (FG) located at the distal end. Among FnIII repeats, eight domains are constitutively expressed (Fn1-8), while nine are alternatively spliced (FnA1, FnA2, FnA3, FnA4, FnB, FnAD2, FnAD1, FnC, and FnD). Alternative RNA splicing yields various isoforms of $\mathrm{TnC}$ with different numbers of domains $(7,8)$. Via its structurally distinct domains and by variations in domains resulting from alternative splicing, $\mathrm{TnC}$ is predisposed to interact with different types of cellular receptors or components of the extracellular matrix, thereby generating an impressive functional diversity (9). In the central nervous system, $\mathrm{TnC}$ is widely expressed at early developmental stages (10), being mainly synthesized by immature astrocytes and radial glia during neuronal migration and differentiation (11). TnC stimulates astrocyte proliferation in vitro (12) and contributes to regenerative processes, such as peripheral nerve regeneration and wound healing in the brain (13-15). It also regulates the phenotype of cultured astrocytes in vitro, thus possibly contributing to astrocytic scar formation after spinal cord injury (16). TnC is also known to interact with other extracellular matrix components, including fibronectin $(17,18)$ and chondroitin sulfate proteoglycans $(19,20)$, relevant for regeneration after injury.

Recovery of locomotor functions after spinal cord injury in constitutively TnC-deficient (TnC-/-) mice was found to be reduced when compared with their wild-type $(\mathrm{TnC}+/+)$ littermates. Overexpression of the $\mathrm{FnD}$ domain of $\mathrm{TnC}$ in injured spinal cords improved regeneration (21). In TnC-/mice synaptic rearrangements in the lumbar spinal cord and the H-reflex response were both attenuated after injury when compared with wild-type littermates. It is thus conceivable that TnC exerts its beneficial effects by modifying synaptic responses to injury (21). These findings are in agreement with previous observations that $\mathrm{TnC}$ enhances neurite outgrowth and supports neuronal survival $(22,23)$.

$\mathrm{TnC}$ is transiently expressed in acute inflammation and may contribute to chronic inflammation in pathological conditions (24). $\mathrm{TnC}$ also plays a role in temporal and spatial modulation of inflammation after SCI (25). Since TnC may promote axonal regrowth during acute inflammation, but also contribute to further damage during the chronic phase, investigation of individual $\mathrm{TnC}$ domains at specific times and in specific cell types is much needed. Since after SCI TnC is secreted mainly by astrocytes, we examined the impact of individual $\mathrm{TnC}$ fragments on astrocytic physiology in vitro and in vivo.

TNC-null mice were crucial for discovering the significance of heterophilic and homophilic TNC interactions in glial scar formation. The same mice were instrumental in the initial discovery of positive effects of TNC on regeneration after spinal cord injury (21). We now report on the dissection of functional TNC domains which may allow designing structures of therapeutic value. Results of our present study indicate that gap closure in vitro in an astrocyte scratch assay is delayed by TnC fibronectin-like fragments, mainly due to decreasing astrocyte proliferation. Fragments also upregulated mRNA levels of pro-inflammatory cytokines in astrocyte cell cultures. In spinal cord tissue, addition of $\mathrm{TnC}$ fragments immediately after SCI did not alter total cell proliferation rate. TnC, and in particular fragment $\mathrm{FnD}$ increased the numbers of activated microglia/macrophages 7 days after injury. Altogether results may explain how astrocyte functions are restricted to the border of the injury site to allow microglia/macrophages to form a lesion core during the first stages of glial scar formation through $\mathrm{TnC}$, and in particular FnD.

\section{MATERIALS AND METHODS}

\section{Animals}

Animals used in experiments were wild-type C57BL/6 (TnC+/+) mice and constitutively tenascin- $\mathrm{C}$ deficient $(\mathrm{TnC}-/-)$ mice inbred on the C57BL/6 background for more than 10 generations, and maintained in the Animal Facility of the Faculty of Biology, University of Belgrade. Animals were housed under standard conditions $\left(21 \pm 1{ }^{\circ} \mathrm{C}, 50 \%\right.$ humidity, 12:12 h light/dark cycle, water and food ad libitum). TnC-/- mice were derived from the original colony (26). All experimental procedures complied with the NIH Guide for Care and Use of Laboratory Animals (1985) and the European Communities Council Directive (86/609/EEC) and were approved by the Ethics Committee of the Faculty of Biology, University of Belgrade.

\section{Treatments}

Five recombinantly expressed proteins representing different domains of TnC were studied. We used all alternatively spliced fragments and some of these that are present in all variants of the tenascin- $\mathrm{C}$ molecule. For injury experiments, we used the alternatively spliced $\mathrm{FnD}$ and $\mathrm{FnA}$ fragments, as they were 
shown to be the most promising ones in the in vitro experiments (23) and in a previous in vivo study (21). Alternatively spliced fragments are of special interest as they are upregulated upon injury (27).

Fibronectin type III-like repeats 6-8 (Fn 6-8) and epidermal growth factor-like repeats (EGFL) are constitutively expressed, whereas fibronectin type III-like repeats A, D, and C (FnA, FnD, $\mathrm{FnC}$ ) are generated by alternative splicing. Fragments were generated as described (28). For in vitro experiments, treatment groups were labelled as follows: "SW" for the control group with only scratch wounding performed, "no SW" for noninjured cells, "EGFL", "FnA", "FnC", "FnD”, "Fn6-8”, for groups in which the fragments were individually added after SW, and "Fn $(D+A)$ ", "Fn $(D+A+C)$ " for groups in which a combination of fragments was added. For in vivo experiments, all labels were the same except for the control group, which was named "Injury", indicating that only compression spinal cord injury (SCI) was performed.

\section{Chemicals, Reagents, and Solutions}

Acrylamide/Bis-acrylamide, ammonium persulfate (APS), bovine serum albumin (BSA), diethyl pyrocarbonate (DEPC), chloroform, EDTA, glucose, glycerol, Mowiol embedding medium, NP 40, paraformaldehyde (PFA), poly-L-lysine (PLL), sodium dodecyl sulphate (SDS), tetramethyl ethylenediamine (TEMED), Tris base, Triton ${ }^{\mathrm{TM}} \mathrm{X}-100$, trypsin, Tween $20, \beta-$ mercaptoethanol, $\mathrm{NaCl}, \mathrm{Na}_{2} \mathrm{HPO}_{4}, \mathrm{NaHCO}_{3}, \mathrm{NaOH}, \mathrm{HCl}$, $\mathrm{KH}_{2} \mathrm{PO}_{4}, \mathrm{KCl}$ were from Sigma-Aldrich (St. Louis, Missouri, USA). Leibovitz's L-15 medium, penicillin/streptomycin, foetal bovine serum (FBS), Dulbecco's modified Eagle's medium (DMEM) were from Gibco (Thermo Fisher Scientific, USA). TRIzol reagent, $\mathrm{H}_{2} \mathrm{O}$, Power SYBR $^{\mathrm{TM}}$ Green PCR Master Mix were from Invitrogen (Thermo Fisher Scientific, USA). High Capacity cDNA Reverse Transcription Kit was from Applied Biosystems (Thermo Fisher Scientific, USA). 4,6-Diamidino-2phenylindole (DAPI) was from Molecular Probes (Thermo Fisher Scientific, USA). Protease/phosphatase inhibitor cocktail, Pierce micro BCA Protein Assay Kit, and PAGE ruler were from Thermo Fisher Scientific, USA. Clarity ECL Substrate was from BioRad Laboratories (Hercules, CA, USA). Ethanol, isopropanol, and methanol were from Zorka Pharma (Šabac, Serbia).

\section{Cortical Astrocyte Culture}

Primary cell cultures were prepared as described (29). Briefly, for each genotype cortices from three mice, 0 to 2 days old, of both sexes were pooled. Tissue was mechanically dissociated and then centrifuged twice at 500xg for $5 \mathrm{~min}$. Before the third centrifugation, the cell suspension was passed successively $3-5$ times through $21 \mathrm{G}$ ( $0.8 \mathrm{~mm}$ ) and 23G (ø0.6 mm) needles. All steps were performed in Leibowitz L-15 isolating medium supplemented with $100 \mathrm{IU} / \mathrm{ml}$ penicillin, $0.1 \mathrm{mg} / \mathrm{ml}$ streptomycin, and $0.1 \%$ BSA. Finally, cells were resuspended in growth medium (Dulbecco's modified Eagle medium-low glucose, DMEM, supplemented with 10\% foetal bovine serum, D-glucose to a final concentration of $25 \mathrm{mmol} / \mathrm{L}$, $100 \mathrm{IU} / \mathrm{ml}$ penicillin, and $100 \mu \mathrm{g} / \mathrm{ml}$ streptomycin), seeded in a $60 \mathrm{~mm}$ Petri dish and maintained in a humidified atmosphere of 5\%
$\mathrm{CO}_{2} / 95 \%$ air at $37^{\circ} \mathrm{C}$. The culture medium was replaced every 2 to 3 days. Upon reaching confluency, cells were trypsinized $(0.25 \%$ trypsin and $0.02 \%$ EDTA) and seeded in new Petri dishes. Once confluence was reached again, cells were seeded according to the particular experimental design. Three cell cultures per genotype were prepared for all in vitro experiments.

\section{Scratch Wound Assay}

For the scratch wound assay (SW), astrocytes were seeded in $35 \mathrm{~mm}$ Petri dishes at a density of $2 \times 10^{4}$ cells $/ \mathrm{cm}^{2}$ and maintained until complete confluency as described $(30,31)$. Monolayers were scratched with a sterile $200 \mu \mathrm{l}$ pipette tip, followed by addition of fresh culture medium containing $10 \mu \mathrm{g} / \mathrm{ml}$ per TnC fragments. Three to four scratches were made per Petri dish. To ensure imaging of the same fields at different times, a straight line was drawn in the middle of the Petri dish bottom. For each SW, two areas, just above and below the line, were imaged using the AxioObserver A1 inverted microscope (Carl Zeiss GmbH, Germany), EM512 CCD (Digital Camera System, Evolve, Photometrics), and 10× (A-Plan) objective. Cells were imaged immediately after scratching and addition of fragments $(0 \mathrm{~h})$, and later at $6,12,24$, and $48 \mathrm{~h}$. For each image a gap border was selected and the gap area $\left(\mu \mathrm{m}^{2}\right)$ was determined using the ImageJ software package. Relative wound closure was calculated with the formula:

$$
\text { Relative woundclosure }=\left[A\left(t_{0}\right)-A(t)\right] / A\left(t_{0}\right)
$$

where A represents wound area determined at a given time point $t$.

\section{Immunocytochemistry}

For immunolabeling, cells were maintained on PLL-coated glass coverslips (ø $15 \mathrm{~mm}$ ). Twenty-four $\mathrm{h}$ after scratching and treatment with $\mathrm{TnC}$ fragments, cells were fixed in $4 \%$ formaldehyde for $20 \mathrm{~min}$ at room temperature (RT). After several washes with phosphate-buffered saline (PBS), cells were permeabilized with $0.1 \%$ Triton in PBS for 15 min and blocked with a solution containing 5\% BSA in PBS for $1 \mathrm{~h}$, at RT. Following the overnight incubation with primary rabbit antiKi67 (1:500, Abcam, ab15580, RRID: AB_443209) or rabbit antiGFAP (1:500, DAKO, Z0334, RRID: AB_10013382) antibodies in $1 \% \mathrm{BSA}$ in PBS at $4^{\circ} \mathrm{C}$, cells were washed and incubated with secondary donkey anti-rabbit AlexaFluor-555 antibodies for $2 \mathrm{~h}$, at RT and in the dark (1:200, Invitrogen A-31572, RRID: AB_162543). Nuclei were stained with DAPI (1:4,000, for $15 \mathrm{~min}$, at RT), and the glass coverslips were mounted on microscope slides with MOWIOL solution. Micrographs along the SW were acquired using AxioObserver A1 inverted microscope (Carl Zeiss GmbH, Germany), EM512 CCD (Digital Camera System, Evolve, Photometrics), and 10× (APlan) objective. Omission of primary antibodies did not show immunoreactivity. Images were quantified using ImageJ software. Proliferation rate was defined as the proportion of $\mathrm{Ki67}+$ nuclei within the total number of DAPI+ nuclei. GFAP immunoreactivity was quantified and presented as the corrected total cell fluorescence (CTCF) of integrated density, calculated 
for each frame after using the "Rolling ball" background subtraction method in ImageJ software.

\section{Western Blot Analysis}

Astrocytes seeded in $60 \mathrm{~mm}$ Petri dishes were scratched and treated with $\mathrm{TnC}$ fragments. Twenty-four $\mathrm{h}$ later, cultures were washed with pre-heated PBS, mechanically detached by scraping, collected in ice-cold PBS, and centrifuged for $5 \mathrm{~min}$ at $500 \times \mathrm{g}$. The pellet was resuspended in $500 \mu \mathrm{l}$ of ice-cold RIPA lysis buffer, supplemented with $0.5 \% \mathrm{w} / \mathrm{v}$ protease inhibitor cocktail. Subsequently, lysates were centrifuged for $10 \mathrm{~min}$ at $10,000 \times \mathrm{g}$ and $4^{\circ} \mathrm{C}$. The supernatant was collected and the protein concentration was determined using the BCA protein assay kit, according to the manufacturer's instruction. Samples (5 $\mu$ g protein) were mixed with $6 \times$ Laemmle sample buffer $(375 \mathrm{mM}$ Tris- $\mathrm{HCl}, \mathrm{pH}$ 6.8, $12 \%$ SDS, $60 \% \mathrm{w} / \mathrm{v}$ glycerol, and $0.03 \%$ bromophenol blue). Proteins were resolved on $12 \%$ SDS-PAGE gels and electrotransferred to a PVDF support membrane (Immobilon-P transfer membrane, Millipore, Merck, Germany). Membranes were blocked with 5\% BSA in Tris buffered saline/Tween 20 (TBST) and incubated overnight at $4^{\circ} \mathrm{C}$ with primary rabbit anti-GFAP antibodies (1:7,000, DAKO, Z0334, RRID: AB_10013382), followed by a $2 \mathrm{~h}$ incubation with secondary HRP-conjugated donkey antirabbit antibody (1:10,000, Santa Cruz, sc-2305, AB_641180). Bands were visualized with ECL solution and Chemi Doc-It imaging system (UVP, Upland, CA, USA). Membranes were then subjected to Abcam mild stripping protocol: two times in stripping buffer for $10 \mathrm{~min}$, two times in PBS for $10 \mathrm{~min}$, and two times in TBST for $5 \mathrm{~min}$. Membranes were blocked as abovementioned, incubated with mouse anti- $\beta$-actin $(1: 1,000$, Santa Cruz, sc-47778, RRID: AB_2714189) overnight at $4^{\circ} \mathrm{C}$, followed by secondary HRP-conjugated donkey anti-mouse antibody (1:10,000, Santa Cruz, sc-2096, RRID: AB_641168). Bands were visualized as stated above, and quantified using ImageJ software. The measured optical density of GFAP immunoreactivity was normalized to the corresponding optical density of $\beta$-actin bands serving as a loading control.

\section{mRNA Isolation and Real-Time PCR}

Astrocytes were seeded in 6-well plates at $2 \times 10^{4}$ cells $/ \mathrm{cm}^{2}$ density. After reaching confluence, the cultures were subjected to SW and treated with $\mathrm{TnC}$ fragments. Six hours later, sample lysates were collected using TRIzol reagent and the total RNA was subjected to phenol/chloroform extraction and ethanol precipitation (32). Since it has been shown that the median half-life of mRNA for all genes in mammals is up to $7 \mathrm{~h}$, after which time mRNA is less stable/decays (33), we decided that $6 \mathrm{~h}$ would be the most appropriate time point to analyze the effects of fragments and also to not lose the information due to various signaling convergence and mRNA decay. The $6 \mathrm{~h}$ mRNA expression usually correlates with the protein abundance peak occurring 12-24 h later, which was confirmed in the present case. RNA concentrations were determined by measuring the absorbance at $260 \mathrm{~nm}$ and the purity was estimated from 260/ $280 \mathrm{~nm}$ and 260/230 nm ratios. For the synthesis of cDNA, $1 \mu \mathrm{g}$ of total RNA was used. The real-time PCR reaction mixture contained $2 \mu \mathrm{l}$ cDNA, $5 \mu \mathrm{l}$ QTM SYBR Green PCR Master Mix,
$0.5 \mu \mathrm{l}$ of both reverse and forward primers $(100 \mathrm{pmol} / \mu \mathrm{l})$, and $2 \mu \mathrm{l}$ RNase-free water. Amplification was carried out under the following conditions: $10 \mathrm{~min}$ of enzyme activation at $95^{\circ} \mathrm{C}, 40$ cycles of $15 \mathrm{~s}$ denaturation at $95^{\circ} \mathrm{C}, 30 \mathrm{~s}$ annealing at $64^{\circ} \mathrm{C}, 30 \mathrm{~s}$ amplification at $72^{\circ} \mathrm{C}$, and $5 \mathrm{~s}$ fluorescence measurements at $72^{\circ} \mathrm{C}$. The amplification and product detection were carried out with QuantStudioTM 3 Real-Time PCR System (Applied Biosystems, Foster City, CA, USA). Relative gene expression is presented as a $\log _{2}$-fold change of mRNA expression. $\beta$-actin was used as a housekeeping molecule. Primer sequences are listed in Table $\mathbf{1 .}$

\section{Spinal Cord Injury}

Compression spinal cord injury (SCI) was performed on 10- to 12 -week-old mice as described (21). In short, before surgery, animals were anesthetized by intraperitoneal injections of ketamine and xylazine (100 and $5 \mathrm{mg} / \mathrm{kg}$ body weight, respectively, both from Sigma-Aldrich). Laminectomy was carried out at the T7-T9 level using mouse laminectomy forceps (Fine Science Tools, Foster City, California, USA). Then, the exposed spinal cord was compressed for $1 \mathrm{~s}$ using a custom-made device that consisted of watchmaker forceps mounted on a stereotaxic frame and driven by an electromagnetic device. Immediately after the injury, fragments $\mathrm{FnD}, \mathrm{FnA}$, their combination $(300 \mu \mathrm{g} / \mathrm{ml}$ per treatment), or the vehicle control $(0.9 \% \mathrm{NaCl}$ in water) were injected into the lesion site. Injections into areas surrounding the injury site were also carried out $1 \mathrm{~mm}$ rostrally and caudally to the lesion site. Next, the skin was closed with a 3-0 silk suture (Ethicon, Somerville, New Jersey, USA). Mice were then kept on heating pads at $35^{\circ} \mathrm{C}$ for $24 \mathrm{~h}$ to prevent hypothermia and caged individually in a temperaturecontrolled room $\left(22^{\circ} \mathrm{C}\right)$ on soft bedding with softened rodent chow and water within reach ad libitum. Bladders were manually voided twice a day. Animals were sacrificed 7 days later.

Four animals per group [vehicle control, FnA, FnD, Fn(D+A)] per each genotype (32 mice in total) underwent spinal cord injury. Criteria for the exclusion of mice from the study were, per ethical permit, weight loss of more than $20 \%$ over $24 \mathrm{~h}$, passive position of animal in the cage, the lack of reaction to the experimenter, overall poor general condition as seen by the animal being immobile, cold with wet hair, and signs of severe urinary infection. Three animals were excluded for these reasons.

\section{Tissue Preparation and Immunohistochemistry}

Mice were perfused transcardially under anesthesia with saline $(0.9 \% \mathrm{NaCl})$ for $30 \mathrm{~s}$, followed by perfusion with $4 \%$ formaldehyde in $0.1 \mathrm{M}$ phosphate buffer for $10 \mathrm{~min}$. The spinal cord was then removed through double laminectomy

TABLE 1 | List of primer pairs for real-time PCR.

\begin{tabular}{lll}
\hline $\begin{array}{l}\text { Target } \\
\text { gene }\end{array}$ & \multicolumn{1}{c}{ Forward } & \multicolumn{1}{c}{ Reverse } \\
\hline GFAP & CGGAGACGCATCACCTCTG & TGGAGGAGTCATTCGAGACAA \\
TNF- $\alpha$ & CTGAACTCGGGGTATCGG & GGCTTGTACTCGAATITGAGA \\
I-1 $\beta$ & AAAAGCCTCGTGCTGTCGGACC & TTGAGGCCCAAGGCACAGGT \\
$\beta$-actin & GGGCTATGCTCTCCCTCAC & GATGTCACGCACGATTCC
\end{tabular}


and placed in $4 \%$ formaldehyde for $2 \mathrm{~h}$ at $4^{\circ} \mathrm{C}$. After postfixation, tissue was cryoprotected in $0.1 \mathrm{M}$ phosphate buffer supplemented with $30 \%$ sucrose at $4^{\circ} \mathrm{C}$ overnight and then frozen at $-80^{\circ} \mathrm{C}$ until further use. Sagittal serial sections $(25 \mu \mathrm{m}$ thick) were cut using a LeicaCM 1850 cryostat (Leica Microsystems, Wetzlar, Germany), and sections were collected on SuperFrost ${ }^{\circledR}$ Plus slides (Menzel-Gläser, Braunschweig, Germany). After rehydration in PBS, sagittal spinal cord sections were covered with $0.1 \%$ glycine in PBS for $10 \mathrm{~min}$ at RT. Blocking with $10 \%$ bovine serum albumin (BSA) and $0.1 \%$ Triton X-100 was performed for $45 \mathrm{~min}$ at RT. Primary antibodies were diluted in $2 \% \mathrm{BSA}$ in PBS and kept on the tissue sections overnight at $4^{\circ} \mathrm{C}$. The following primary antibodies were used: mouse anti-GFAP (1:400, DAKO, Z0334), goat anti-Ibal (1:300, Abcam) and rabbit anti-Ki67 (1:200, Abcam). After several washes in PBS, secondary antibodies diluted in PBS with 2\% BSA were incubated for $2 \mathrm{~h}$ in the dark at RT. Secondary antibodies were donkey anti-mouse Alexa 488 (1:200, Invitrogen), donkey anti-goat Alexa 488 (1:200, Invitrogen), and donkey anti-rabbit Alexa 555 (1:200, Invitrogen). After washing with $\mathrm{PBS}$, slides were incubated with TO-PRO 3 (1:40,000; Thermo Ficher Scientific T3605) to counterstain nuclei. Sections were rinsed with PBS and mounted in MOWIOL medium (Sigma Aldrich).

\section{Confocal Imaging, Analysis of Proliferation and GFAP Immunoreactivity in Tissue Sections}

Cell proliferation and GFAP immunoreactivity at the injury site were analyzed in spinal cords of three animals per group [Injury only, Injury plus FnA, FnD, or Fn(D+A)] in both genotypes. Five sections per spinal cord were examined. A confocal laser microscope (LSM 510, Carl Zeiss, Jena, Germany) equipped with $488 \mathrm{~nm}$ Argon, 555 and $633 \mathrm{~nm}$ HeNe lasers was used for obtaining images. Z-stacks were obtained in the visible zone of the injury using oil-immersion Plan Neofluar 40x 1.3 NA objective. For proliferation rates, results were expressed as the portion of Ki67+ nuclei in total DAPI+ numbers in cell cultures or TO-PRO-3 stained nuclei in tissue sections using ImageJ (Rasband, W.S., ImageJ, National Institutes of Health, Bethesda, Maryland, USA, https://imagej.nih.gov/ij/, 1997-2018.). GFAP immunoreactivity was quantified and is presented as the corrected total cell fluorescence (CTCF) of integrated density, calculated for each frame after using the "Rolling ball" background subtraction method in ImageJ software.

\section{Stereological Analysis}

The density of microglial cells in spinal cord tissue sections was obtained by stereological analysis as described (34). Counting was carried out with an Axio Imager. M1 microscope (Carl Zeiss) equipped with a motorized stage and a Stereo Investigator 9 software-controlled microscope system (MicroBrightField). First, a low-power magnification (10× objective) was used to outline the injury region and equally sized rostral and caudal regions next to it which extended for $\leq 1,500 \mu \mathrm{m}$ on both sides of the injury site. In both genotypes, spinal cords from three animals were analyzed for each group (Injury, FnD). Six sections ( $25 \mu \mathrm{m}$, every 10 serial section) per spinal cord were investigated. Cells were counted based on Ibal immunoreactivity and DAPI fluorescence and classified as activated microglia if they exhibited a polygonal shape or as resting if they showed a branched morphology. The following parameters were set: guard space depth, $2 \mu \mathrm{m}$, base and height of the dissector, $60 \times 60 \mu \mathrm{m}$, and $10 \mu \mathrm{m}$; distance between the optical dissectors, $180 \mu \mathrm{m}$; objective $20 \times$ Plan-Neofluar 20×/0.50.

\section{Statistical Analysis}

Two-way ANOVA was used to determine the effects of treatment and genotype regarding the examined parameters in cultures or tissue sections. Three-way ANOVA was used to analyze the effect of treatment, genotype and sampling position (injury, rostral or caudal) on the density of microglia. Statistically significant interactions, simple main effects, and pairwise comparisons were determined. All pairwise comparisons were run for each simple main effect with $95 \%$ confidence intervals and p-values Bonferroni-adjusted within each simple main effect. All computations were performed using the SPSS 20 software package (SPSS Inc., Chicago, IL, USA). Data from each experiment are summarized as a box and whisker plot and shown as mean \pm SD throughout the Results section. Values for $\mathrm{p}$ within the range of $0.001-0.05$ are given as precise numbers, whereas values lower than 0.001 are presented as $<0.001$.

\section{RESULTS}

\section{Tenascin-C Fragments Attenuate Gap Closure In Vitro}

A scratch wound assay was performed in TnC+/+ and TnC-/cortical astrocyte cultures to measure the response to mechanical injury (30). It is worth mentioning that mechanical stretching of an astrocyte monolayer is not the most adequate model for assaying glial scar formation since it lacks many components of in vivo injury (35). Unlike in tissues where astrocytes are confined to the lesion border, they are the only cell type in cultures.

First, we assessed whether the rate of gap closure differs between the two genotypes upon the addition of different $\mathrm{TnC}$ fragments. Cells were monitored at $0,6,12,24$, and $48 \mathrm{~h}$ after scratching (see an example of Image J measurements in Figure 1A). Zero $\mathrm{h}$ served as a reference for closure calculation, and by $48 \mathrm{~h}$ all gaps were closed regardless of treatment. As described (36) and observed in our present study, the most informative time point is $24 \mathrm{~h}$ after scratching, allowing for evaluation of fragment application, astrocytic activation, and stable progression of the astrocytic front (Figure 1B). Quantifications of other time points with the exception of 0 and $48 \mathrm{~h}$ are given as stacked bar charts (Supplementary Figure 1). Representative images of the gap area of the same frame taken at 0 and $24 \mathrm{~h}$ in control group (SW) and groups treated with $\mathrm{FnA}, \mathrm{FnD}$, and $\mathrm{Fn}(\mathrm{D}+\mathrm{A})$ are shown in Figure 1C. 
A

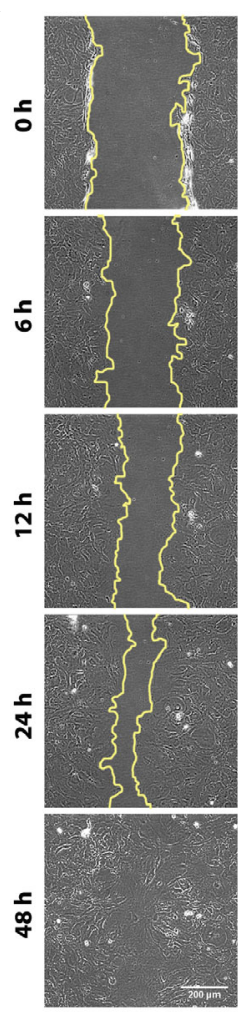

B

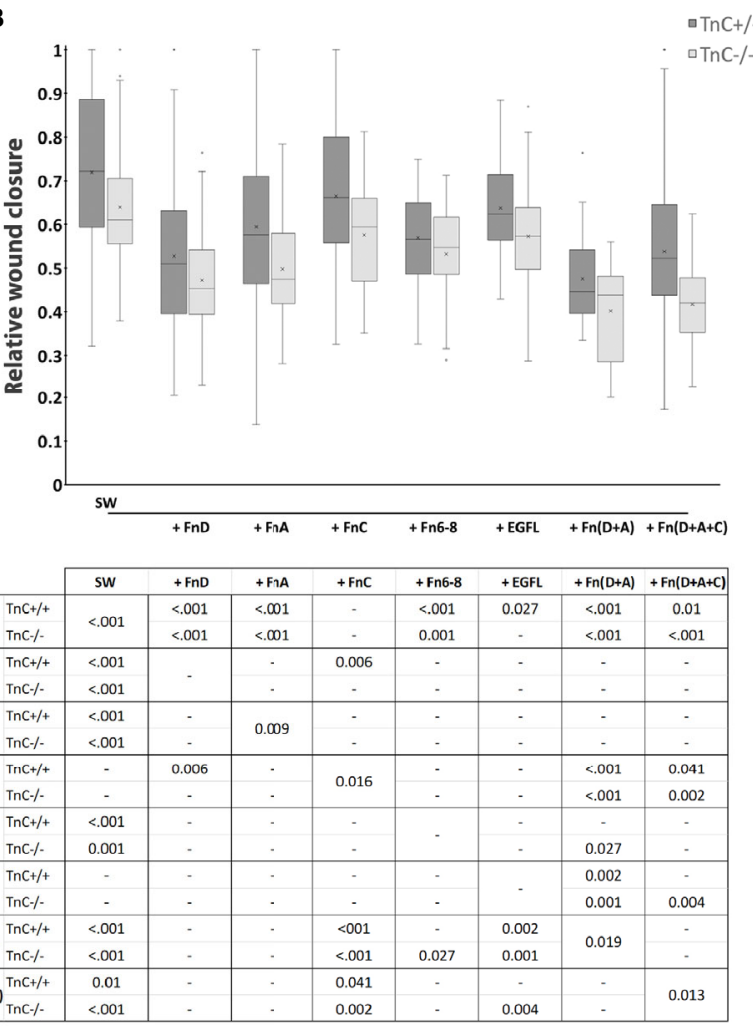

C

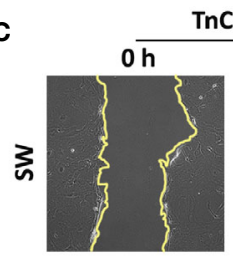

$\mathrm{TnC}+/+$
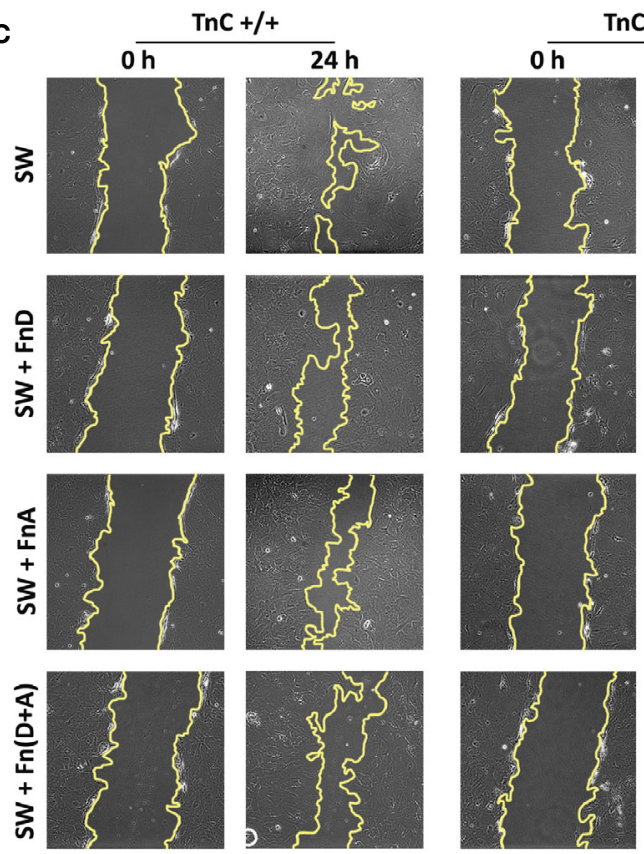

TnC - /-
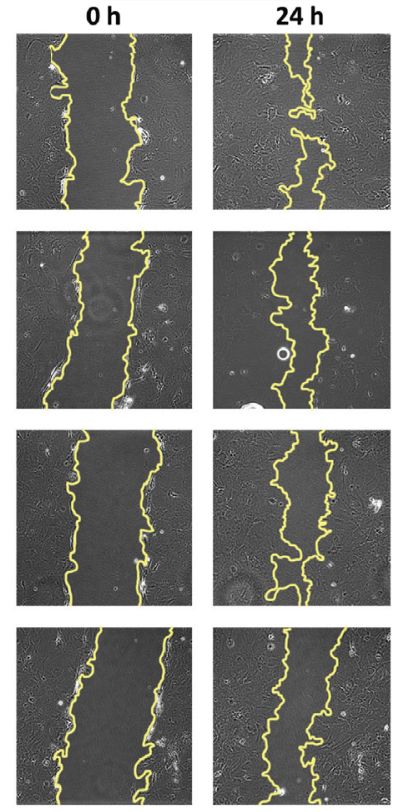

FIGURE 1 | TnC fragments attenuate wound closure in cultured astrocytes. (A) Representative brightfield images of five time points of the gap area measured in the ImageJ program. (B) Relative wound closure is presented in a box-and-whisker plot indicating the impact of $\mathrm{TnC}$ fragments on gap closure in $\mathrm{TnC}+/+$ and $\mathrm{TnC}-/-$ cortical astrocyte cultures after $24 \mathrm{~h}$. Two-way ANOVA analysis shows no interaction between genotype and treatment, but both the effects of treatment and genotype were significantly different $(p<0.001)$. The most potent effect was produced by FnA, FnD, and combinations Fn(D+A) and Fn(D+A+C) in both genotypes. All pairwise significance comparisons are given in the table below the box-and-whisker plot. $n=3$ independent astrocyte culture preparations. (C) Representative images of the gap at 0 and $24 \mathrm{~h}$ after scratching in the control group (SW) and groups treated with $\mathrm{FnA}, \mathrm{FnD}$, and Fn(D+A). 
Both the effects of treatment and genotype were significant $(p<0.001)$. The rate of injury closure reached the highest values in the control $\mathrm{TnC}+/+$ group, with application of fragments retarding this process. Compared to the control group (SW), addition of FnA, FnD, Fn6-8, EGFL, Fn(D+A), and Fn(D+A+C) decreased the gap closure $[p<0.001$ for FnA, FnD, Fn6-8, $\mathrm{Fn}(\mathrm{D}+\mathrm{A}) ; \mathrm{p}=0.027$ for EGFL; $\mathrm{p}=0.010$ for $\mathrm{Fn}(\mathrm{D}+\mathrm{A}+\mathrm{C})]$. Fragments FnC and EGFL did not affect gap closure. FnD decreased gap closure compared to FnC $(p=0.006)$. Combination $\mathrm{Fn}(\mathrm{D}+\mathrm{A})$ slowed down the gap closure compared to FnC $(p<0.001)$ and EGFL $(p<0.001)$. Similarly, Fn $(D+A+C)$ decreased gap closure compared to $\mathrm{FnC}(p=0.041)$. In cultures of TnC-/- mice, where gap closure was most pronounced in the control group, fragments FnA, FnD, Fn6-8, Fn(D+A), and Fn $(D+A+C)$ reduced gap closure $[p<0.001$ for $F n A, F n D, F n(D+A)$, $\mathrm{Fn}(\mathrm{D}+\mathrm{A}+\mathrm{C}) ; \mathrm{p}=0.010$ for Fn6-8], while FnC and EGFL did not affect gap closure. The combined fragments $\mathrm{Fn}(\mathrm{D}+\mathrm{A})$ also reduced gap closure when compared to FnC, Fn6-8, and EGFL ( $p<0.001, p=0.027, p<0.001$, respectively), while combination $\mathrm{Fn}(\mathrm{D}+\mathrm{A}+\mathrm{C})$ showed lower closure rates than FnC $(p<0.001)$ and EGFL $(p=0.004)$. In TnC $+/+$ cultures, gap closure was more pronounced than in TnC-/- cultures $(p<0.001)$. FnD and Fn6-8 attenuated gap closure in both genotypes, while FnA, FnC, Fn $(\mathrm{D}+\mathrm{A}+\mathrm{C})$ attenuated gap closure in TnC-/- genotype (FnA: $p=$ 0.009; FnC: $p=0.016 ; \mathrm{D}+\mathrm{A}+\mathrm{C}: p=0.013)$. Mean values \pm standard deviations for each treatment and genotype are shown in Table 2.

\section{Tenascin-C Fragments Reduce Proliferation Rate In Vitro}

Migration and proliferation are two main processes that contribute to gap closure after scratching. Previous data show that the rate of astrocyte migration after scratching is the same as in non-injured, low density cultures used as a control group, whereas proliferation increases, making only proliferation an injury-specific response (30). On the basis of these observations, we assessed the proliferation rate (Ki67+/DAPI+ numbers) $24 \mathrm{~h}$ after scratching and application of fragments (Figures 2A, B). In this experiment another treatment group was added with no scratching and addition of fragments (no SW). There was a significant interaction between the effects of genotype and treatment on cell proliferation rate $(p=0.005)$ with both the effects of genotype and treatment being significant $(p=0.040, p<0.001)$.

In $\mathrm{TnC}+/+$ cultures the highest rate of proliferation was observed in the control SW group, while the addition of all fragments or their combinations decreased it $[p<0.001$ for EGFL, FnA, FnC, FnD, Fn6-8, Fn(D+A), Fn(D+A+C)]. EGFL, FnA, FnD, Fn6-8, Fn $(D+A), F n(D+A+C)$ reduced proliferation compared to FnC $[p<0.001$, except for $\mathrm{Fn}(\mathrm{D}+\mathrm{A}+\mathrm{C}), p=0.004]$. $\mathrm{Fn}(\mathrm{D}+\mathrm{A}+\mathrm{C})$ led to a higher proliferation rate compared to
FnD $(p=0.012)$, FnA $(p=0.001)$, Fn6-8 $(p<0.001)$, and EGFL $(p=0.001)$. The no SW group exhibited a higher proliferation rate than other treatments $(p<0.001)$, but was not different from the SW group.

Similarly, in the TnC-/- group the highest rate of proliferation was observed in the SW group, while the addition of all fragments or their combinations reduced it $[p<0.001$ for EGFL, FnA, FnC, FnD, Fn6-8, Fn(D+A), Fn(D+A+C)]. Compared to $\mathrm{FnC}$, the other treatments reduced the proliferation rate [EGFL, FnA, FnD, Fn6-8, Fn(D+A) and Fn(D+A+C), $p<$ 0.001 for all]. Fragment FnA additionally reduced the proliferation rate compared to EGFL $(p=0.039)$. No SW group had a lower proliferation rate than the SW group $(p<0.001)$, but a higher proliferation rate than all other treatment groups ( $p<0.001$ for all) except for FnC. Comparisons within each treatment, between the two genotypes, showed a significant difference only in SW ( $p=$ $0.007)$, FnC $(p=0.020)$, Fn6-8 $(p=0.052)$, EGFL $(p<0.001)$ groups, where higher proliferation rates were observed in TnC-/cultures. Mean values \pm standard deviations for each treatment and genotype are shown in Table 3.

\section{GFAP Expression is Upregulated in Tenascin-C-/- Astrocyte Cultures Regardless of Fragment Addition}

GFAP, as a cytoskeletal marker protein of astrocytes, is upregulated after scratching (37). TnC also affects the levels of GFAP in lesion-activated astrocytes in vitro (38). Therefore, we examined Gfap mRNA (Figure 3A) and protein levels (Figures 3B-D) upon SW and treatment with TnC fragments (6 and $24 \mathrm{~h}$, respectively).

Only the genotype's effect on Gfap mRNA levels was significant $(p<0.001)$. Gfap mRNA expression levels in TnC -/showed a $\log _{2}$-fold increase versus the $\mathrm{TnC}+/+$ in the $\mathrm{SW}$ group. Compared to $\mathrm{TnC}+/+$ cultures, upregulation of TnC-/- Gfap mRNA levels was most pronounced in the no SW group (TnC+/+ vs TnC-/-: $1.061 \log _{2}$-fold change, $p=0.041$ ) and in the FnC treatment group (TnC+/+ vs TnC-/-: $1.180 \log _{2}$-fold change), while $\mathrm{Fn}(\mathrm{D}+\mathrm{A})$ only tended to show upregulation (TnC+/+ vs TnC-/-: $0.680 \log _{2}$-fold change, not significant).

GFAP protein expression was measured by Western blot analysis $24 \mathrm{~h}$ after SW and application of fragments (Figure 3B). Representative images of GFAP (48 kDa) and $\beta$-actin (42 $\mathrm{kDa}$ ) are in Figure 3C. Only the genotype's effect on protein levels was significant $(p<0.001)$. GFAP was more expressed in the no SW group in TnC-/- cultures compared to $\mathrm{TnC}+/+$ cultures (no SW: $2.15 \pm 1.14$; no SW: $0.97 \pm 0.30, p<0.001$ ). Scratch wounding and treatments resulted in GFAP protein upregulation in TnC-/- cultures compared to the $\mathrm{TnC}+/+$ cultures in the SW, FnD, FnA, and FnC groups $(p<0.001 ; p=0.003$;

TABLE 2 | Mean value \pm standard deviation for relative gap closure.

\begin{tabular}{|c|c|c|c|c|c|c|c|c|}
\hline & sw & +EGFL & $+\mathrm{FnA}$ & $+\mathrm{FnC}$ & $+F n D$ & +Fn6-8 & $+F n(D+A)$ & $+F n(D+A+C)$ \\
\hline $\mathrm{TnC}+/+$ & $0.74 \pm 0.19$ & $0.64 \pm 0.12$ & $0.59 \pm 0.19$ & $0.66 \pm 0.16$ & $0.53 \pm 0.19$ & $0.57 \pm 0.11$ & $0.48 \pm 0.11$ & $0.54 \pm 0.20$ \\
\hline TnC -/- & $0.64 \pm 0.12$ & $0.57 \pm 0.13$ & $0.50 \pm .12$ & $0.58 \pm 0.11$ & $0.47 \pm 0.11$ & $0.53 \pm 0.11$ & $0.40 \pm 0.10$ & $0.42 \pm 0.09$ \\
\hline
\end{tabular}


A

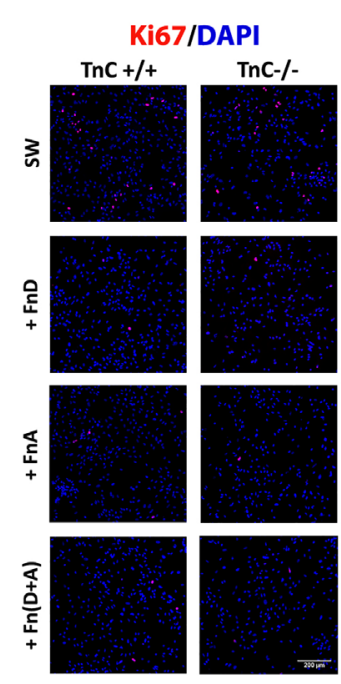

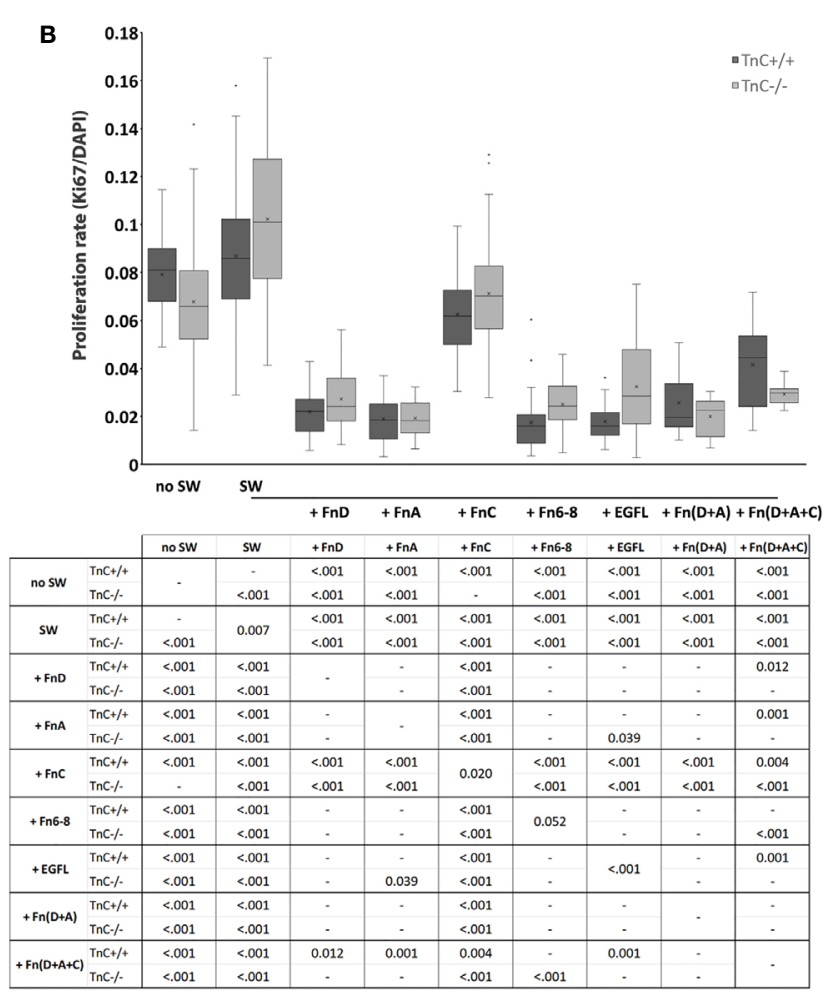

FIGURE 2 | ThC fragments reduce proliferation in the astrocyte scratch wound assay. (A) Representative micrographs of Ki67+/DAPI+ immunofluorescence at 24 h after scratching in the control group and groups treated with FnA, FnD, and Fn(D+A); bar: $200 \mu$ m. (B) Proliferation was calculated as the number of Ki67+ nuclei compared to total DAPI+ nuclei. Results are presented as a box-and-whisker plot. Two-way ANOVA analysis shows a statistically significant interaction between the effects of genotype and treatment on cell proliferation rate $(p=0.005)$ with both the effects of genotype and treatment being significant $(p=0.040, p<0.001$, respectively). A statistically significant decrease in proliferation is seen in the presence of $F n A$, FnD, and Fn(D+A). All statistically significant pairwise comparisons are displayed below the box-and-whisker plot. $n=3$ independent astrocyte cultures.

$p=0.002 ; p=0.001$, respectively). Immunostaining was performed to visually confirm Western blot GFAP protein expression levels (Figure 3D).

\section{Pro-Inflammatory Tnf- $\alpha$ and IL-1 $\beta$ Levels are Upregulated by FnD, FnA, Fn6-8, and EGFL in Both Genotypes}

It has been reported that $\mathrm{TnC}$ upregulates pro-inflammatory cytokine production in different cell types although there are no data available for astrocytes (39). Cytokine production in astrocyte cultures after scratching and addition of $\mathrm{TnC}$ fragments was tested in terms of mRNA levels of Tnf- $\alpha, I l-1 \beta$, Il-6, and Il-10, with Il-6 and Il-10 not being detectable. rtPCR results for Tnf- $\alpha$ mRNA levels showed that the treatment effect was significant $(p<0.001)$ (Figure 4A). In TnC $+/+$ cultures, addition of EGFL, FnA, FnD, and Fn6-8 fragments upregulated Tnf- $\alpha$ mRNA expression compared to SW, compared to the no
SW group and compared to the FnC group. Similarly, in TnC-/cultures, EGFL, FnA, FnD and Fn6-8 fragments increased Tnf- $\alpha$ mRNA levels compared to SW, no SW and FnC treatment group. In the case of $I L-1 \beta$ mRNA expression (Figure 4B) the effect of the treatment was significant $(p<0.001)$. Application of fragments EGFL, FnA, FnD and Fn6-8 upregulated $I l-1 \beta$ mRNA levels in $\mathrm{TnC}+/+$ cultures compared to the SW group $(p<0.001$ for FnA and FnD; $p=0.001$ for EGFL and Fn6-8), no SW ( $p<0.001$ for EGFL, FnA, FnD, Fn6-8) and FnC $(p=0.001$ for FnA and FnD; $p=0.003$ for EGFL and Fn6-8). Similarly, in TnC-/- cultures, EGFL, FnA, FnD and Fn6-8 increased $I l-1 \beta$ mRNA expression when compared to SW $(p=0.001$ for EGFL and FnA; $p<0.001$ for FnD and Fn6-8), no SW $(p<0.001$ for EGFL, FnA, FnD, Fn6-8) and FnC; $(p<0.001$ for EGFL, FnA, FnD, Fn6-8). Mean values \pm standard deviations for levels of proinflammatory cytokines TNF- $\alpha$ and IL-1 $\beta$ in astrocyte culture are presented in Tables 4 and 5, respectively.

TABLE 3 | Mean values \pm standard deviations of proliferation rate in the astrocyte scratch wound assay.

\begin{tabular}{|c|c|c|c|c|c|c|c|c|c|}
\hline & no SW & sw & +EGFL & $+F n A$ & $+\mathrm{FnC}$ & $+F n D$ & +Fn6-8 & $+F n(D+A)$ & $+F n(D+A+C)$ \\
\hline $\mathrm{TnC}+/+$ & $0.079 \pm 0.015$ & $0.087 \pm 0.026$ & $0.018 \pm 0.008$ & $0.019 \pm 0.010$ & $0.062 \pm 0.016$ & $0.022 \pm 0.010$ & $0.017 \pm 0.012$ & $0.026 \pm 0.013$ & $0.042 \pm 0.017$ \\
\hline TnC-/- & $0.078 \pm 0.032$ & $0.098 \pm 0.032$ & $0.032 \pm 0.020$ & $0.019 \pm 0.007$ & $0.071 \pm 0.023$ & $0.027 \pm 0.011$ & $0.025 \pm 0.011$ & $0.020 \pm 0.008$ & $0.029 \pm 0.004$ \\
\hline
\end{tabular}



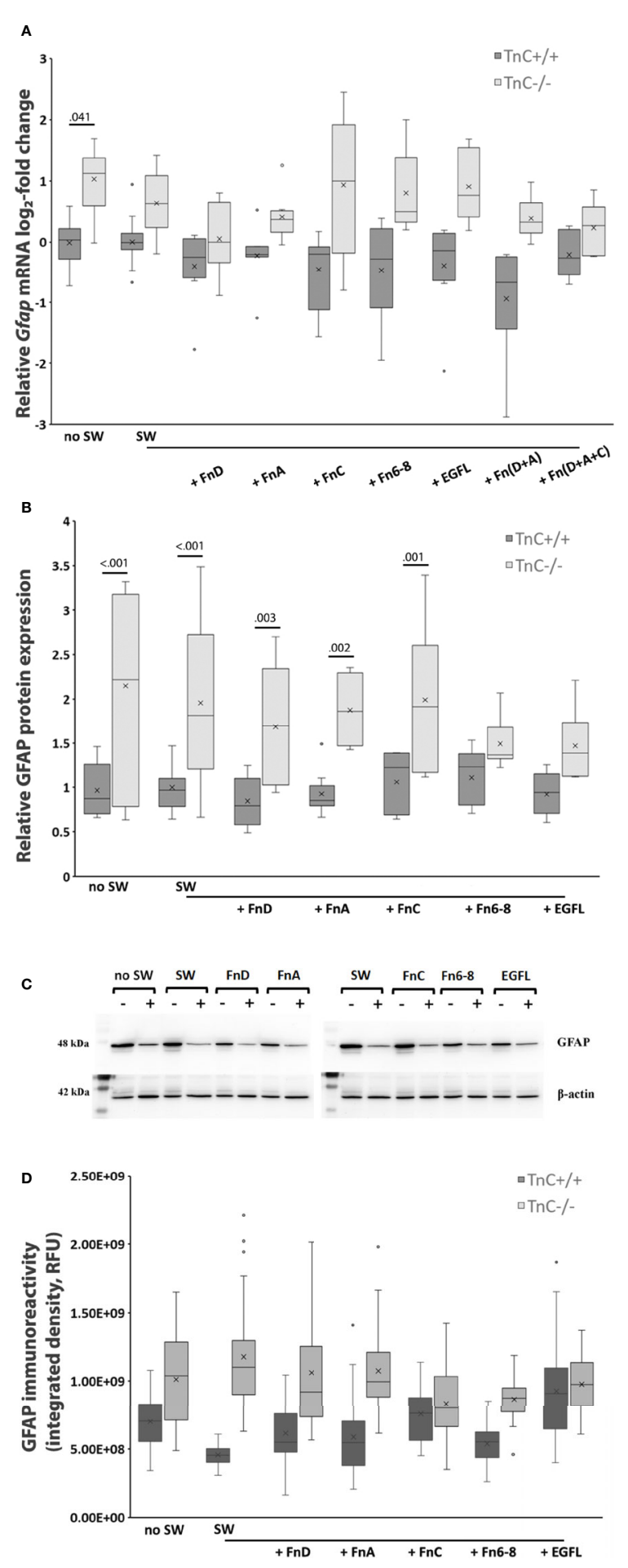

FIGURE 3 | GFAP expression is upregulated in TnC-/- astrocyte cultures both in the absence and presence of fragments. (A) Relative log ${ }_{2}$-fold change of Gfap mRNA expression $6 \mathrm{~h}$ after scratching and fragment addition is shown in a box-and-whisker plot. Two-way ANOVA shows a genotype effect ( $p<0.001)$. Gfap mRNA was significantly upregulated in TnC-/- no SW group compared to its TnC+/+ counterpart $(p=0.041) . n=4$ independent astrocyte culture preparations. (B) Relative GFAP protein expression as estimated by Western blot analysis $24 \mathrm{~h}$ after scratching and application of fragments. Results are presented as box-andwhisker plot. Two-way ANOVA shows statistical significance of genotype $(p<0.001)$. GFAP is more expressed in the no SW group of TnC-/- versus TnC+/+

cultures $(p<0.001)$. Scratching alone and FnA, FnC, and FnD application lead to upregulation of GFAP protein levels in TnC-/- versus TnC+/+ astrocytes $(p<0.001$; $p=0.003 ; p=0.002: p=0.001$, respectively). $\mathrm{n}=3$ independent astrocyte culture preparations. (C) Representative images of Western blots for GFAP (48 kDa) and $\beta$-actin (42 kDa). (D) GFAP immunoreactivity is presented as Integrated density box-and-whisker plot. $\mathrm{n}=1$ astrocyte culture. 
A
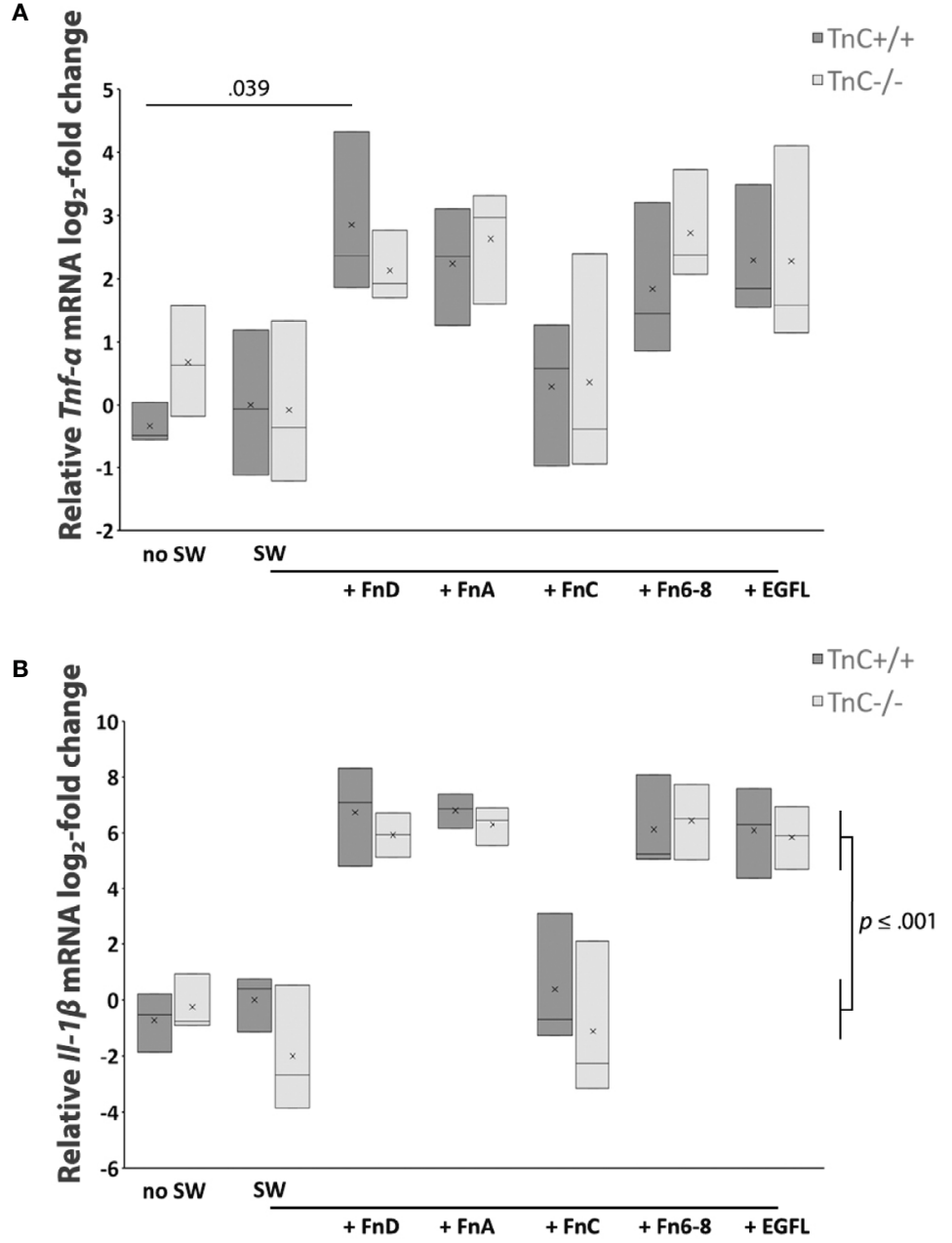

FIGURE 4 | Pro-inflammatory cytokines TNF- $\alpha$ and IL-1 $\beta$ are upregulated by FnD, FnA, Fn6-8, and EGFL fragments in astrocyte cultures of both genotypes. (A) Relative $\log _{2}$-fold change of Tnf- $\alpha$ mRNA expression $6 \mathrm{~h}$ after scratching and fragment addition is presented in a box-and-whisker plot. Two-way ANOVA shows that only the treatment effect was significantly different $(p<0.001)$. In both TnC+/+ and TnC-/- genotypes, FnD, FnA, Fn6-8, and EGFL upregulate Tnf- $\alpha$ mRNA levels ( $2 \log _{2}$-fold change for all conditions) versus the no SW, SW, and FnC groups. Only the FnD fragment statistically upregulates Tnf- $\alpha$ mRNA levels versus the no SW in TnC+/+ cultures $(p=0.039)$. $n=3$ independent astrocyte culture preparation. (B) Relative $\log _{2}$-fold change of $I I-1 \beta \mathrm{mRNA}$ levels $6 \mathrm{~h}$ after scratching and fragment addition as presented in the box-and-whisker plot. Two-way ANOVA shows that only the treatment was statistically significant $(p<0.001)$. In TnC+/+ and TnC-/- cultures, addition of EGFL, FnA, FnD, and Fn6-8 upregulates $\|-1 \beta$ mRNA levels $\left(\sim 7 \log _{2}\right.$-fold change, $p \leq 0.001$ for all conditions) versus levels of no SW, SW, and FnC. $n=3$ independent astrocyte cultures.

\section{Tenascin-C Affects Astrocytes and Microglia/Macrophages in Injured Spinal Cords}

In vivo glial scar formation was induced by compression SCI. Evaluation was performed 7 days after the injury when inflammation is in its early phase and thus can be modulated via different mechanisms (40).

Proliferation in vivo was estimated in the same way as in vitro and in reference to all cell types in the injury region (Figures $\mathbf{5 A}$, B). Ki67 immunolabeling indicated higher levels of proliferating cells within the region of injury and a decrease of such cells

TABLE 4 | Mean values \pm standard deviations of proinflammatory cytokine TNF- $\alpha$ in astrocyte cultures.

\begin{tabular}{lccccrrr}
\hline & no SW & SW & +EGFL & +FnA & +FnC & +FnD & +Fn6-8 \\
\hline $\mathrm{TnC}+/+$ & $-0.33 \pm 0.32$ & $0.00 \pm 1.16$ & $2.29 \pm 1.04$ & $2.24 \pm 0.92$ & $0.29 \pm 1.15$ & $2.85 \pm 1.30$ & $1.84 \pm 1.22$ \\
$\mathrm{TnC}-/-$ & $0.68 \pm 0.88$ & $-0.08 \pm 1.30$ & $2.28 \pm 1.60$ & $2.63 \pm 0.91$ & $0.36 \pm 1.79$ & $2.13 \pm 0.56$ & $2.72 \pm 0.88$
\end{tabular}


TABLE 5 | Mean values \pm standard deviations of proinflammatory cytokine IL-1 $\beta$ levels in astrocyte cultures.

\begin{tabular}{|c|c|c|c|c|c|c|c|}
\hline & no SW & sw & +EGFL & $+\mathrm{FnA}$ & $+\mathrm{FnC}$ & $+F n D$ & +Fn6-8 \\
\hline $\mathrm{TnC}+/+$ & $-0.73 \pm 1.05$ & $0.00 \pm 0.99$ & $6.09 \pm 1.61$ & $6.81 \pm 0.61$ & $0.37 \pm 2.37$ & $6.73 \pm 1.78$ & $6.12 \pm 1.70$ \\
\hline TnC-/- & $-0.25 \pm 1.02$ & $-2.01 \pm 2.27$ & $5.84 \pm 1.13$ & $6.30 \pm 0.68$ & $-1.12 \pm 2.81$ & $5.92 \pm 0.79$ & $6.43 \pm 1.35$ \\
\hline
\end{tabular}

further away, in all groups (Figure 5A). Even though two-way ANOVA showed that the overall effect of fragment application was significant $(p=0.043)$, pairwise comparisons revealed no significant difference (Figure 5B).
Quantification of GFAP immunoreactivity (Figure 5C) in the injury region revealed a statistically significant effect of genotype $(p<0.001)$, as well as a statistically significant interaction of genotype and treatment effects $(p=0.034)$. Addition of fragment

A
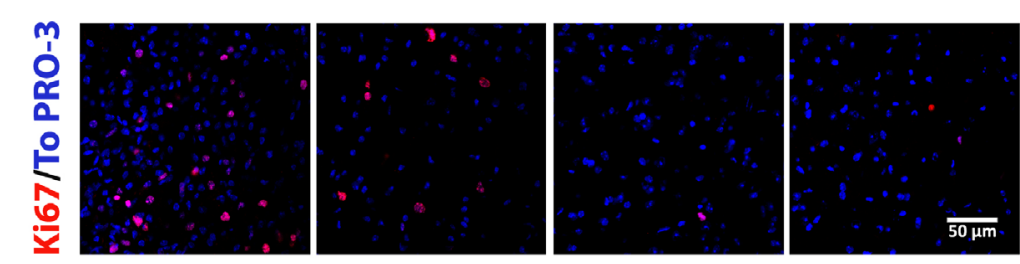

Injury site

B

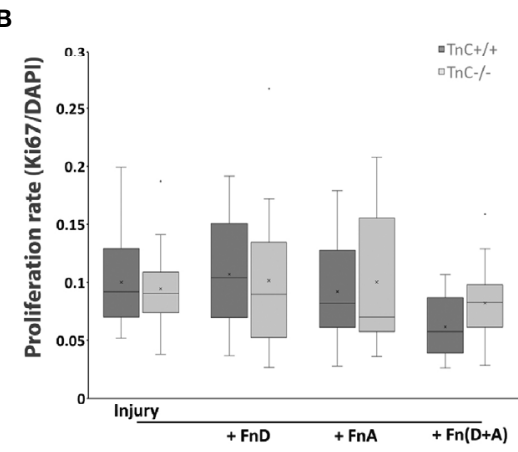

D

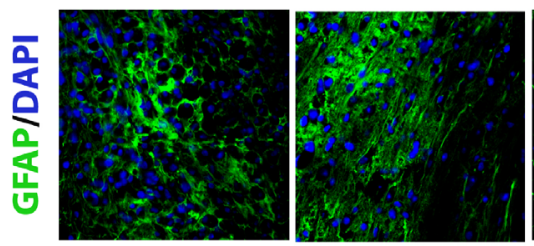

Injury site
C

.


FnD to TnC-/- mice decreased GFAP immunoreactivity compared to $\mathrm{FnD}$ in TnC+/+ animals $(p<0.001)$. This effect was also observed with combined $\mathrm{Fn}(\mathrm{D}+\mathrm{A})$ treatment $(p=$ 0.002). Representative images of GFAP immunofluorescence at the injury site of spinal cord treated with FnD and Fn(D+A) fragments in both genotypes are presented in Figure 5E. In TnC-/- mice, although FnD reduced GFAP immunoreactivity compared to FnA, only a trend was observed $(p=0.061)$. Change in the morphology of GFAP positive astrocytes in the injury region compared to the more rostral or caudal regions in the spinal cord was also observed (Figure 5D). Astrocytes within the injury region formed a honeycomb-like structure, characteristic of the glial scar (41), whereas more distally they exhibited the fibrous quiescent phenotype (Figure 5D). Mean values \pm standard deviations of GFAP immunoreactivity at the injury region are presented in Table 6.

Another typical glial scar element, especially within the first week after injury, are microglia/macrophages $(40,42)$. Whereas astrocyte processes overlap at the site of injury and cell bodies are difficult to distinguish individually, activated microglia/ macrophages are recognized as distinct polygonal cells, being framed by astrocytes (Figures 6A). Thus, the density of activated and resting microglia/macrophages could be quantified throughout the injury region and surrounding rostral and caudal regions. The overall effects of genotype and sampling position on the density of activated microglia were significant $(p<0.001)$, as well as their interaction in the injury group $(p=$ 0.032) (Figure 6B). The density of activated microglia/ macrophages within the injury site was higher in $\mathrm{TnC}+/+$ mice in both injury and $\mathrm{FnD}$ groups compared to other regions $(p<$ 0.001). In FnD- treated TnC-/- mice, the density of activated microglia/macrophages in the injury region was higher than in rostral $(p=0.003)$ or caudal $(p<0.001)$ regions. When normalized to the total microglia/macrophage number, the proportion of activated microglia was higher in the injury site compared to rostral and to caudal positions in all of the conditions $(p<0.001)$ reaching the highest value in the $\mathrm{TnC}+/+$ injury group (Figure 6C). Total microglial cell numbers were

TABLE 6 | Mean values \pm standard deviations of GFAP immunoreactivity at the injury site of the spinal cord.

\begin{tabular}{|c|c|c|c|c|}
\hline & Injury & + FnA & + FnD & $+F n(D+A)$ \\
\hline $\mathrm{TnC}+/+$ & $1.16 \mathrm{E}^{+06} \pm 7.67 \mathrm{E}^{+05}$ & $1.41 \mathrm{E}^{+06} \pm 9.47 \mathrm{E}^{+05}$ & $1.61 \mathrm{E}^{+06} \pm 8.03 \mathrm{E}^{+05}$ & $1.67 \mathrm{E}^{+06} \pm 8.79 \mathrm{E}^{+05}$ \\
\hline $\mathrm{TnC}-/-$ & $1.06 \mathrm{E}^{+06} \pm 5.95 \mathrm{E}^{+05}$ & $1.19 \mathrm{E}^{+06} \pm 7.01 \mathrm{E}^{+05}$ & $5.87 \mathrm{E}^{+05} \pm 5.42 \mathrm{E}^{+05}$ & $8.21 \mathrm{E}^{+05} \pm 4.46 \mathrm{E}^{+05}$ \\
\hline
\end{tabular}

A
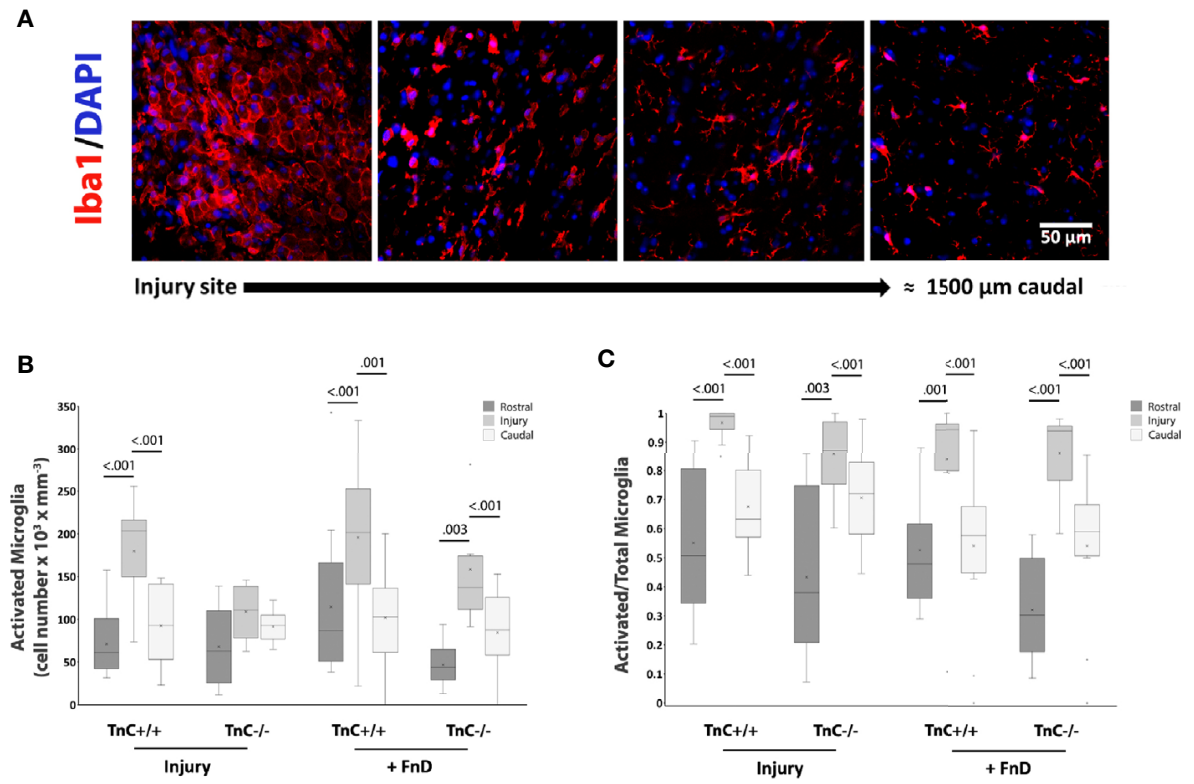

FIGURE 6 | ThC affects activation of microglia in the injury region. (A) Representative images of Iba1+ and DAPI immunostaining show activated, polygonal microglia framed by astrocytes, at the injury site, and a more quiescent microglia phenotype further away. Calibration bar for all panels: 50 um. (B) Density of activated microglia by stereo-investigator analysis at the injury region and surrounding rostral and caudal regions of equal size 7 days after injury. Results are shown as a boxand-whisker plot. The effects of genotype and sampling position on the density of activated microglia are significant $(p<0.001)$, as well as their interaction in the Injury group $(p=0.032)$ as analyzed by three-way ANOVA. The density of activated microglia in $\mathrm{TnC}+/+$ mice in the injury region is higher in $\mathrm{TnC}+/+$ mice in both Injury and FnD groups compared to the other sampling positions $(p<0.001)$. FnD application in TnC-/- mice increases the density of activated microglia at the injury region more so than rostrally $(p=0.003)$ or caudally $(p<0.001$. (C) A box-and-whisker plot showing Activated/(Activated + Resting) microglia numbers per region. The proportion of activated microglia is higher at the injury site than rostrally and caudally in the injury and FnD groups of both genotypes $(p<0.001)$. $n=3$ animals per group for each genotype. 
calculated as the sum of activated and resting microglia. Resting microglia density was a complementary image of activated microglia density (Supplementary Figure S2). Average numbers \pm standard deviations of activated microglia in the spinal cord injury site of $\mathrm{TnC}+/+$ and $\mathrm{TnC}-/$ - mice are presented in Table 7, and mean values \pm standard deviations of the portion of activated microglia out of total microglia numbers is presented in Table 8.

\section{DISCUSSION}

We studied the effects of $\mathrm{TnC}$ and its fragments on the glial reaction to injury in vitro and in vivo. In the context of an astrocyte scratch assay, we found that, independent of the genotype, TnC fragments attenuated gap closure. FnD and its combination with the fragment FnA were the most effective. Proliferation of astrocytes in vitro was decreased upon application of alternatively spliced FnD, FnA, FnC, their combinations and constitutively expressed Fn6-8 and EGFL fragments. These results suggest that fragments delay gap closure through decreased astrocyte proliferation.

Previous observations indicate that astrocyte proliferation is enhanced in a scratch wound assay (30), while TnC is known to reduce proliferation of adult human astrocytes in vitro (16). Alternatively spliced region encompassing FnIII repeats from A to D binds to the cell surface annexin II receptor (43), which inhibits cell migration in the process of gap closure (44). A smaller, $190 \mathrm{kDa}$ isoform of $\mathrm{TnC}$, comprising only the constitutively expressed domains, binds to contactin/F11 through FnIII repeats (45) and promotes cell adhesion (46). FnIII domain can also bind to the $\beta$ subunit of integrin receptors $(47,48)$ and affect both cell migration and proliferation through reorganization of the actin cytoskeleton. $(39,49)$

A scratch wound assay elicits in vitro a response of a single cell type, such as astrocytes, to mechanical injury only, with astrocytes tending to close the gap through migration and proliferation. On the other hand, in vivo, injury includes mechanical and chemical stages, as well as heterogenous cell types, disruption of the blood brain barrier and inflammation, all contributing to a more complex reaction during glial scar formation. Seven days after SCI, pro-inflammatory cells form the lesion core, while astrocytes reside on the border of the lesion site, initially providing protection of the surroundings, and eventually forming the scar tissue (40).

Levels of pro-inflammatory cytokines Tnf- $\alpha$ and $I l-1 \beta$ were increased in vitro in astrocytes upon addition of EGFL, FnA, FnD, and Fn6-8 fragments, regardless of the genotype. TnC has three main binding partners in inflammatory signalling: Toll-like receptor 4 (TLR4), integrins $\alpha 9 \beta 1$ and $\alpha \mathrm{V} \beta 3$. When TLR4 is activated by $\mathrm{TnC}$, the most frequent outcome is the production of soluble pro-inflammatory mediators, such as IL-6, TNF- $\alpha$ and IL-1 $\beta$ by various cell types (39). These cytokines are upregulated also in peritoneal macrophages via activation by $\mathrm{TnC}$ of integrin $\alpha \mathrm{V} \beta 3$ and NFKB signaling pathways (49).

In our experiments, TnC-/- astrocyte cultures exhibited consistently higher GFAP protein and mRNA expression levels, regardless of the type of fragment used for treatment. As already reported, the GFAP expression level was higher in spinal cords of TnC-/- embryos, which is not seen thereafter in adult spinal cords of non-injured mice (38). In our study, in vivo, 7 days after SCI, GFAP immunoreactivity was the same in $\mathrm{TnC}+/+$ and TnC-/- mice treated with vehicle or FnA after injury. Injection of $\mathrm{FnD}$ or $\mathrm{Fn}(\mathrm{D}+\mathrm{A})$ led to a difference between the two genotypes, with higher levels in $\mathrm{TnC}+/+$ than in $\mathrm{TnC}-/-$ mice (40).

In vivo proliferation at the injury site of the spinal cord was the same in $\mathrm{TnC}+/+$ and TnC-/- mice 7 days after SCI. Since in vitro proliferation of astrocytes decreased upon fragment application, regardless of the genotype, it is thus possible that FnD and FnA induce proliferation of cell types other than astrocytes or that $\mathrm{TnC}$ does not affect cell proliferation in the tissue during this time frame after injury. We propose that this difference is due to different binding partners on the surface of different cell types.

$\mathrm{TnC}$ had been implicated in regulating production of proinflammatory cytokines/chemokines, chemotaxis and phagocytosis through the interaction with TLR4 in cultures of microglia (50). Our results on the microglia/macrophage response to injury showed that the FnD fragment increases the number of activated microglia/macrophages at the injury site.

TABLE 7 | Average numbers \pm standard deviations of activated out of total microglia in the injured spinal cord of $\mathrm{TnC}+/+$ and $\mathrm{TnC}-/-$ mice $\left(\right.$ cell number $\left.\times 10^{3} \times \mathrm{mm}^{-3}\right)$.

\begin{tabular}{|c|c|c|c|c|c|c|}
\hline & \multicolumn{3}{|c|}{ Injury } & \multicolumn{3}{|c|}{$+F n D$} \\
\hline & Rostral & Injury & Caudal & Rostral & Injury & Caudal \\
\hline $\mathrm{TnC}+/+$ & $92.35 \pm 42.79$ & $179.70 \pm 55.40$ & $71.16 \pm 39.33$ & $101.69 \pm 61.12$ & $195.49 \pm 80.70$ & $114.49 \pm 90.27$ \\
\hline TnC-/- & $91.44 \pm 16.33$ & $108.94 \pm 30.62$ & $67.95 \pm 45.39$ & $84.31 \pm 47.43$ & $158.27 \pm 63.73$ & $46.56 \pm 23.80$ \\
\hline
\end{tabular}

TABLE 8 | Mean values \pm standard deviations of the portion of activated microglia numbers in the injured spinal cord of ThC $+/+$ and ThC $-/-$ mice.

\begin{tabular}{|c|c|c|c|c|c|c|}
\hline & \multicolumn{3}{|c|}{ Injury } & \multicolumn{3}{|c|}{+ FnD } \\
\hline & Rostral & Injury & Caudal & Rostral & Injury & Caudal \\
\hline $\mathrm{TnC}+/+$ & $0.68 \pm 0.14$ & $0.97 \pm 0.05$ & $0.55 \pm 0.23$ & $0.54 \pm 0.27$ & $0.84 \pm 0.24$ & $0.53 \pm 19$ \\
\hline TnC-/- & $0.71 \pm 0.15$ & $0.86 \pm 0.13$ & $0.43 \pm 0.28$ & $0.54 \pm 0.24$ & $0.86 \pm 0.14$ & $0.32 \pm 0.17$ \\
\hline
\end{tabular}


Matrix metalloproteases (MMP) induce TnC turnover (46) cleaving it mostly within the alternatively spliced domains, a process through which they also generate soluble fragments which might have different functions than the whole $\mathrm{TnC}$ protein. In the present study, the FnD fragment was singled out as the most potent domain in mediating TnC effects on glial cells within the first week after SCI. Our results may explain how the astrocyte reaction is delayed and restricted to the border of the injury site to allow microglia/macrophages to form a lesion core during the first stages of glial scar formation through action of $\mathrm{TnC}$ and, in particular, FnD. Since this stage is generally regarded as a potential window for therapeutical approaches, modulation of TnC isoforms and their interaction partners could be considered to be valuable targets. Further research is needed to dissect the heterophilic interaction partners which mediate the effects of TnC fragments on astrocytes and microglia/ macrophages upon injury.

\section{DATA AVAILABILITY STATEMENT}

The original contributions presented in the study are included in the article/Supplementary Material. Further inquiries can be directed to the corresponding authors.

\section{ETHICS STATEMENT}

The animal study was reviewed and approved by the Ethics Committee of the Faculty of Biology, University of Belgrade.

\section{AUTHOR CONTRIBUTIONS}

$\mathrm{DB}$ performed the in vitro experiments, assisted in the in vivo experiment data analysis, and wrote the manuscript. MA assisted in the in vitro experiments, data analyses, and manuscript writing. MP assisted in the in vivo experiments, immunohistochemistry, and data analysis. IJ conceptualized and design the study, performed the in vivo experiments, and wrote the manuscript. EF provided study consultation and wrote the manuscript.

\section{REFERENCES}

1. Oyinbo CA. Secondary injury mechanisms in traumatic spinal cord injury: a nugget of this multiply cascade. Acta Neurobiol Experimentalis (2011) 71 (2):281-99.

2. Bradbury EJ, Burnside ER. Moving beyond the glial scar for spinal cord repair. Nat Commun (2019) 10(1):3879. doi: 10.1038/s41467-019-11707-7

3. Windle WF, Clemente CD, Chambers WW. Inhibition of formation of a glial barrier as a means of permitting a peripheral nerve to grow into the brain. J Comp Neurol (1952) 96(2):359-69. doi: 10.1002/cne.900960207

4. Anderson MA, Burda JE, Ren Y, Ao Y, O'Shea TM, Kawaguchi R, et al. Astrocyte scar formation aids central nervous system axon regeneration. Nature (2016) 532(7598):195-200. doi: 10.1038/nature17623

5. Zhang Y, Winterbottom JK, Schachner M, Lieberman AR, Anderson PN. Tenascin- $\mathrm{C}$ expression and axonal sprouting following injury to the spinal dorsal columns in the adult rat. J Neurosci Res (1997) 49(4):433-50. doi: 10.1002/(SICI)1097-4547(19970815)49:4<433::AID-JNR5>3.0.CO;2-9
MS provided encouragement for the study concept, envisioned the importance of tenascin- $\mathrm{C}$ domains, provided study consultation, and wrote the manuscript. PA provided the study concept and design, consultation, and wrote the manuscript. All authors contributed to the article and approved the submitted version.

\section{FUNDING}

The study was funded by the DAAD/MESTD project "Involvement of Tenascin-C in Astrocyte Scarring After Spinal Cord Injury" (45103-01766/2014-09/6) and the Ministry of Education, Science and Technological Development of the Republic of Serbia, contract number: 451-03-68/2020-14/200178.

\section{ACKNOWLEDGMENTS}

The authors thank Dr. Djordje Miljkovic for help with real-time PCR experiments, Dr Jelena Katić for help with the Stereo Investigator and Dr. Gabriele Loers for help with the production of fragments.

\section{SUPPLEMENTARY MATERIAL}

The Supplementary Material for this article can be found online at: https://www.frontiersin.org/articles/10.3389/fimmu.2020.624612/ full\#supplementary-material

Supplementary Figure 1 | Gap closure in astrocyte cultures of $\mathrm{TnC}+/+$ and TnC-/mice in the presence of different $\mathrm{TnC}$ fragments. Combined clustered stacked column chart displaying the relative wound closure at different time points after scratching and application of TnC fragments. Stacked columns represent the mean values of relative wound closure \pm SD. $n=3$ independent astrocyte culture preparations.

Supplementary Figure 2 | Density of resting microglia within the spinal cord injury region in $\mathrm{TnC}+/+$ and $\mathrm{TnC}-/-$ mice. Box plots show the density of resting microglia within the injury region, as well as in the surrounding rostral and caudal areas obtained 7 days after the spinal cord injury. Iba1 immunolabeling was assessed by stereological analysis. $n=3$ animals per group for each genotype.
6. Taylor HC, Lightner VA, Beyer WFJr., McCaslin D, Briscoe G, Erickson HP. Biochemical and structural studies of tenascin/hexabrachion proteins. J Cell Biochem (1989) 41(2):71-90. doi: 10.1002/jcb.240410204

7. Nies DE, Hemesath TJ, Kim JH, Gulcher JR, Stefansson K. The complete cDNA sequence of human hexabrachion (Tenascin). A multidomain protein containing unique epidermal growth factor repeats. J Biol Chem (1991) 266 (5):2818-23. doi: 10.1016/S0021-9258(18)49920-6

8. Spring J, Beck K, Chiquet-Ehrismann R. Two contrary functions of tenascin: dissection of the active sites by recombinant tenascin fragments. Cell (1989) 59(2):325-34. doi: 10.1016/0092-8674(89)90294-8

9. Jones FS, Jones PL. The tenascin family of ECM glycoproteins: structure, function, and regulation during embryonic development and tissue remodeling. Dev Dynamics Off Publ Am Assoc Anatomists (2000) 218 (2):235-59. doi: 10.1002/(SICI) 1097-0177(200006)218:2<235::AIDDVDY2>3.0.CO;2-G

10. Bartsch S, Bartsch U, Dörries U, Faissner A, Weller A, Ekblom P, et al. Expression of tenascin in the developing and adult cerebellar cortex. J Neurosci 
Off J Soc Neurosci (1992) 12(3):736-49. doi: 10.1523/JNEUROSCI.12-0300736.1992

11. Götz M, Bolz J, Joester A, Faissner A. Tenascin-C synthesis and influence on axonal growth during rat cortical development. Eur J Neurosci (1997) 9 (3):496-506. doi: 10.1111/j.1460-9568.1997.tb01627.x

12. Ikeshima-Kataoka H, Saito $S$, Yuasa S. Tenascin-C is required for proliferation of astrocytes in primary culture. Vivo (Athens Greece) (2007) 21(4):629-33.

13. Brodkey JA, Laywell ED, O’Brien TF, Faissner A, Stefansson K, Dörries HU, et al. Focal brain injury and upregulation of a developmentally regulated extracellular matrix protein. J Neurosurg (1995) 82(1):106-12. doi: 10.3171/ jns.1995.82.1.0106

14. Irintchev A, Salvini TF, Faissner A, Wernig A. Differential expression of tenascin after denervation, damage or paralysis of mouse soleus muscle. J Neurocytol (1993) 22(11):955-65. doi: 10.1007/BF01218353

15. Martini R. Expression and functional roles of neural cell surface molecules and extracellular matrix components during development and regeneration of peripheral nerves. J Neurocytol (1994) 23(1):1-28. doi: 10.1007/BF01189813

16. Holley JE, Gveric D, Whatmore JL, Gutowski NJ. Tenascin C induces a quiescent phenotype in cultured adult human astrocytes. Glia (2005) 52 (1):53-8. doi: 10.1002/glia.20231

17. Chiquet-Ehrismann R. Anti-adhesive molecules of the extracellular matrix. Curr Opin Cell Biol (1991) 3(5):800-4. doi: 10.1016/0955-0674(91)90053-2

18. Chung CY, Zardi L, Erickson HP. Binding of tenascin-C to soluble fibronectin and matrix fibrils. J Biol Chem (1995) 270(48):29012-7. doi: 10.1074/ jbc.270.48.29012

19. Chiquet M, Fambrough DM. Chick myotendinous antigen. II. A novel extracellular glycoprotein complex consisting of large disulfide-linked subunits. J Cell Biol (1984) 98(6):1937-46. doi: 10.1083/jcb.98.6.1937

20. Vaughan L, Huber S, Chiquet M. Winterhalter KH. A major, six-armed glycoprotein from embryonic cartilage. EMBO J (1987) 6(2):349-53. doi: 10.1002/j.1460-2075.1987.tb04761.x

21. Chen J, Joon Lee H, Jakovcevski I, Shah R, Bhagat N, Loers G, et al. The extracellular matrix glycoprotein tenascin-C is beneficial for spinal cord regeneration. Mol Ther J Am Soc Gene Ther (2010) 18(10):1769-77. doi: $10.1038 / \mathrm{mt} .2010 .133$

22. Lochter A, Schachner M. Tenascin and extracellular matrix glycoproteins: from promotion to polarization of neurite growth in vitro. J Neurosci Off J Soc Neurosci (1993) 13(9):3986-4000. doi: 10.1523/JNEUROSCI.13-0903986.1993

23. Meiners S, Nur-e-Kamal MS, Mercado ML. Identification of a neurite outgrowth-promoting motif within the alternatively spliced region of human tenascin-C. J Neurosci Off J Soc Neurosci (2001) 21(18):7215-25. doi: 10.1523/JNEUROSCI.21-18-07215.2001

24. Goh FG, Piccinini AM, Krausgruber T, Udalova IA, Midwood KS. Transcriptional regulation of the endogenous danger signal tenascin-C: a novel autocrine loop in inflammation. J Immunol (Baltimore Md 1950) (2010) 184(5):2655-62. doi: 10.4049/jimmunol.0903359

25. Gaudet AD, Popovich PG. Extracellular matrix regulation of inflammation in the healthy and injured spinal cord. Exp Neurol (2014) 258:24-34. doi: 10.1016/j.expneurol.2013.11.020

26. Evers MR, Salmen B, Bukalo O, Rollenhagen A, Bösl MR, Morellini F, et al. Impairment of L-type $\mathrm{Ca} 2+$ channel-dependent forms of hippocampal synaptic plasticity in mice deficient in the extracellular matrix glycoprotein tenascin-C. J Neurosci Off J Soc Neurosci (2002) 22(16):7177-94. doi: 10.1523/ JNEUROSCI.22-16-07177.2002

27. Jakovcevski I, Miljkovic D, Schachner M, Andjus PR. Tenascins and inflammation in disorders of the nervous system. Amino Acids (2013) 44 (4):1115-27. doi: 10.1007/s00726-012-1446-0

28. Dörries U, Taylor J, Xiao Z, Lochter A, Montag D, Schachner M. Distinct effects of recombinant tenascin-C domains on neuronal cell adhesion, growth cone guidance, and neuronal polarity. $J$ Neurosci Res (1996) 43(4):420-38. doi: 10.1002/(SICI) 1097-4547(19960215)43:4<420:: AID-JNR4>3.0.CO;2- $\mathrm{H}$

29. Bijelić DD, Milićević KD, Lazarević MN, Miljković DM, Bogdanović Pristov JJ, Savić DZ, et al. Central nervous system-infiltrated immune cells induce calcium increase in astrocytes via astroglial purinergic signaling. $J$ Neurosci Res (2020) 98(11):2317-32. doi: 10.1002/jnr.24699
30. Környei Z, Czirók A, Vicsek T, Madarász E. Proliferative and migratory responses of astrocytes to in vitro injury. J Neurosci Res (2000) 61(4):421-9. doi: 10.1002/1097-4547(20000815)61:4<421::AID-JNR8>3.0.CO;2-4

31. Lampugnani MG. Cell Migration into a Wounded Area In Vitro. In: E Dejana, M Corada, editors. Adhesion Protein Protocols. Totowa, NJ: Humana Press (1999). p. 177-82. doi: 10.1385/1-59259-258-9:177

32. Rio DC, Ares MJr., Hannon GJ, Nilsen TW. Purification of RNA using TRIzol (TRI reagent). Cold Spring Harbor Protoc (2010) 2010(6):pdb.prot5439. doi: $10.1101 /$ pdb.prot5439

33. Sharova LV, Sharov AA, Nedorezov T, Piao Y, Shaik N, Ko MS. Database for mRNA half-life of 19977 genes obtained by DNA microarray analysis of pluripotent and differentiating mouse embryonic stem cells. DNA Res Int J Rapid Publ Rep Genes Genom (2009) 16(1):45-58. doi: 10.1093/dnares/dsn030

34. Jakovcevski I, Siering J, Hargus G, Karl N, Hoelters L, Djogo N, et al. Close homologue of adhesion molecule L1 promotes survival of Purkinje and granule cells and granule cell migration during murine cerebellar development. J Comp Neurol (2009) 513(5):496-510. doi: 10.1002/cne.21981

35. Yoo JY, Hwang CH, Hong HN. A Model of Glial Scarring Analogous to the Environment of a Traumatically Injured Spinal Cord Using Kainate. Ann Rehabil Med (2016) 40(5):757-68. doi: 10.5535/arm.2016.40.5.757

36. Adzic M, Nedeljkovic N. Unveiling the Role of Ecto-5'-Nucleotidase/CD73 in Astrocyte Migration by Using Pharmacological Tools. Front Pharmacol (2018) 9:153. doi: 10.3389/fphar.2018.00153

37. Gao K, Wang CR, Jiang F, Wong AY, Su N, Jiang JH, et al. Traumatic scratch injury in astrocytes triggers calcium influx to activate the JNK/c-Jun/AP-1 pathway and switch on GFAP expression. Glia (2013) 61(12):2063-77. doi: 10.1002/glia.22577

38. Karus M, Denecke B, ffrench-Constant C, Wiese S, Faissner A. The extracellular matrix molecule tenascin $\mathrm{C}$ modulates expression levels and territories of key patterning genes during spinal cord astrocyte specification. Development (2011) 138(24):5321. doi: 10.1242/dev.067413

39. Marzeda AM, Midwood KS. Internal Affairs: Tenascin-C as a Clinically Relevant, Endogenous Driver of Innate Immunity. J Histochem Cytochem Off J Histochem Soc (2018) 66(4):289-304. doi: 10.1369/0022155418757443

40. Beck KD, Nguyen HX, Galvan MD, Salazar DL, Woodruff TM, Anderson AJ. Quantitative analysis of cellular inflammation after traumatic spinal cord injury: evidence for a multiphasic inflammatory response in the acute to chronic environment. Brain J Neurol (2010) 133(Pt 2):433-47. doi: 10.1093/brain/awp322

41. Sun D, Jakobs TC. Structural remodeling of astrocytes in the injured CNS. Neurosci Rev J Bringing Neurobiol Neurol Psychiatry (2012) 18(6):567-88. doi: 10.1177/1073858411423441

42. Zhou X, Wahane S, Friedl MS, Kluge M, Friedel CC, Avrampou K, et al. Microglia and macrophages promote corralling, wound compaction and recovery after spinal cord injury via Plexin-B2. Nat Neurosci (2020) 23 (3):337-50. doi: 10.1038/s41593-020-0597-7

43. Chung CY, Erickson HP. Cell surface annexin II is a high affinity receptor for the alternatively spliced segment of tenascin-C. J Cell Biol (1994) 126(2):53948. doi: $10.1083 /$ jcb.126.2.539

44. Balch C, Dedman JR. Annexins II and V inhibit cell migration. Exp Cell Res (1997) 237(2):259-63. doi: 10.1006/excr.1997.3817

45. Weber P, Ferber P, Fischer R, Winterhalter KH, Vaughan L. Binding of contactin/F11 to the fibronectin type III domains 5 and 6 of tenascin is inhibited by heparin. FEBS Lett (1996) 389(3):304-8. doi: 10.1016/0014-5793 (96)00609-6

46. Giblin SP, Midwood KS. Tenascin-C: Form versus function. Cell Adhesion Migration (2015) 9(1-2):48-82. doi: 10.4161/19336918.2014.987587

47. Giese A, Loo MA, Norman SA, Treasurywala S, Berens ME. Contrasting migratory response of astrocytoma cells to tenascin mediated by different integrins. J Cell Sci (1996) 109( Pt 8):2161-8.

48. Husmann K, Faissner A, Schachner M. Tenascin promotes cerebellar granule cell migration and neurite outgrowth by different domains in the fibronectin type III repeats. J Cell Biol (1992) 116(6):1475-86. doi: 10.1083/ jcb.116.6.1475

49. Shimojo N, Hashizume R, Kanayama K, Hara M, Suzuki Y, Nishioka T, et al. Tenascin- $\mathrm{C}$ may accelerate cardiac fibrosis by activating macrophages via the integrin $\alpha \mathrm{V} \beta 3$ /nuclear factor- $\mathrm{\kappa B} /$ interleukin- 6 axis. Hypertension (Dallas Tex 1979) (2015) 66(4):757-66. doi: 10.1161/HYPERTENSIONAHA.115.06004 
50. Haage V, Elmadany N, Roll L, Faissner A, Gutmann DH, Semtner M, et al. Tenascin C regulates multiple microglial functions involving TLR4 signaling and HDAC1. Brain Behav Immun (2019) 81:470-83. doi: 10.1016/j.bbi.2019.06.047

Conflict of Interest: The authors declare that the research was conducted in the absence of any commercial or financial relationships that could be construed as a potential conflict of interest.
Copyright (ङ 2021 Bijelić, Adžić, Perić, Jakovčevski, Förster, Schachner and Andjus. This is an open-access article distributed under the terms of the Creative Commons Attribution License (CC BY). The use, distribution or reproduction in other forums is permitted, provided the original author(s) and the copyright owner(s) are credited and that the original publication in this journal is cited, in accordance with accepted academic practice. No use, distribution or reproduction is permitted which does not comply with these terms. 